\title{
Low-Dose Dobutamine Cardiovascular Magnetic Resonance Segmental Strain Study of Early Phase of Intramyocardial Hemorrhage Rats
}

\section{Rui Xia}

The First affiliated Hospital of Chongqing Medical University

\section{Bo He}

West China Hospital, Sichuan University

\section{Tong Zhu}

TongJi Hospital, TongJi Medical College, HuaZhong University of Science \& Technology

\section{Yu Zhang}

West China Hospital, Sichuan University

\section{Yushu Chen}

West China Hospital, Sichuan University

\section{Lei Wang}

West China Hospital, Sichuan University

\section{Yang Zhou}

Chongqing Medical University

Jichun Liao

The First affiliated Hospital of Chongqing Medical University

Jie Zheng

Washington University School of Medicine in St. Louis

\section{Yongmei Li}

The First affiliated Hospital of Chongqing Medical University

\section{Fajin Lv}

The First affiliated Hospital of Chongqing Medical University

\section{Fabao Gao ( $\nabla$ gaofabao@yahoo.com )}

West China Hospital, Sichuan University

\section{Research Article}

Keywords: Myocardial infarction, Reperfusion, Magnetic Resonance imaging, Dobutamine

Posted Date: May 25th, 2021

DOl: https://doi.org/10.21203/rs.3.rs-506296/v1 
License: (c) (i) This work is licensed under a Creative Commons Attribution 4.0 International License. Read Full License

Version of Record: A version of this preprint was published at BMC Medical Imaging on November 20th, 2021. See the published version at https://doi.org/10.1186/s12880-021-00709-x. 


\section{Abstract}

Background: This study investigates the segmental myocardial strain of the early phase of intramyocardial hemorrhage (IMH) caused by reperfused myocardial infarction (MI) in rats by low-dose dobutamine (LDD) cardiovascular magnetic resonance (CMR) feature-tracking.

Methods: Nine sham rats and nine rats with 60 -min myocardial ischemia followed by 48 -h reperfusion were investigated using CMR, including T2*-mapping sequence and fast imaging with steady-state precession (FISP) - cine sequence. Another FISP-cine sequence was acquired after 2 min of dobutamine injection; the MI, IMH, and Non-MI (NMI) areas were identified. The values of peak radial strains (PRS) and peak circumferential strains (PCS) of the MI, IMH and NMI segments were acquired. The efficiency of PRS and PCS (EPRS and EPCS, respectively) were calculated on the basis of the time of every single heartbeat.

Results: The PRS, PCS, EPRS, and EPCS of the sham group increased after LDD injection. However, the PRS, PCS, EPRS, and EPCS of the IMH segment did not increase. Moreover, the PRS and PCS of the MI and NMI segments did not increase, but the EPRS and EPCS of these segments increased. The PRS, PCS, EPRS, and EPCS of the IMH segment were lower than those of the MI and NMI segments before and after LDD injection, but without a significant difference between MI segment and NMI segment before and after LDD injection.

Conclusions: LDD could help assess dysfunctions in segments with $\mathrm{IMH}$, especially using the efficiency of strain. IMH was a crucial factor that decreased segmental movement and reserved function.

\section{Background}

Myocardial revascularization reduces the infarction size and the incidence of long-term adverse events in clinical practice. However, myocardial revascularization itself can also lead to intramyocardial hemorrhage (IMH), which has been recognized as a poor prognostic factor in myocardial infarction (MI) for the adverse effect of left ventricular (LV) remodeling [1]. A limited number of studies have demonstrated segmental dysfunctions in the presence of IMH [2-5].

High- and low-dose dobutamine (LDD) stress cardiovascular magnetic resonance (CMR) has been established as an imaging modality with high diagnostic accuracy for evaluating patients with myocardial ischemia [6-7]. Feature-tracking (FT) cardiac magnetic resonance imaging (MRI) is a postprocessing technique that is increasingly being employed to assess global and regional myocardial functions.

In addition, CMR-FT could be used for quantitatively assessing cardiac motion in small animals. Tomas et al. have confirmed the reproducibility of FT in small animals (mice) [8]. Currently, no information exists about the capability of FT for detecting IMH using LDD-CMR in the early phase of reperfused MI rats. 
Hence, this study assesses FT based on LV circumferential strain and radial strain using LDD-CMR and tests its ability to detect segmental dysfunctions in the presence of IMH.

We designed two groups, namely, the reperfused MI group and the sham group, and hypothesized that this MRI strain analysis at rest and with LDD could discriminate IMH, MI, and non-MI (NMI) segments in the reperfusion group and discriminate these segments in the sham group.

\section{Methods}

\section{Animal models}

All experimental procedures were approved by the experimental Animal Ethics Committee of West China Hospital, Sichuan University (Chengdu, China). Nineteen female Sprague-Dawley rats with body weights ranging from 250 to $300 \mathrm{~g}$ were divided into two groups: the reperfusion $(n=10)$ and sham control groups $(n=9)$. Before surgery, the rats were intraperitoneally anesthetized using sodium pentobarbital, and respiration was maintained using a rodent ventilator. A real-time electrocardiogram (ECG) was monitored throughout surgery.

Thoracotomy was performed to introduce the coronary occlusion. The chest was opened at the fourth intercostal space to expose the heart, the pericardium was opened using forceps, and a 6.0 suture was passed underneath the left anterior descending coronary artery at a location $2 \mathrm{~mm}$ proximal to the ostium of the coronary artery. Coronary occlusion was achieved by tightening the suture over a 3.0 suture. The success of occlusion was confirmed by the pale appearance of the myocardial apex area and immediate changes in the ECG profiles, including a significant increase in the amplitude of the QRS complex and STsegment elevation [9].

In the early reperfusion model, all occlusions were maintained for $60 \mathrm{~min}$, followed by reperfusion [10]. Reperfusion was achieved by untying the knot and releasing the suture from occlusion. The success of reperfusion was confirmed by ECG changes including further ST-segment elevation followed by gradual recovery of the ST-segment. In the sham surgery model, no occlusion after thoracotomy was performed.

\section{MRI protocols}

MRI was performed $48 \mathrm{~h}$ after surgery in both groups [10]. All MRI protocols were implemented using a 7.0 T MR system (BRUKER BIOSPEC 70/30). Each rat was anesthetized with isoflurane $(2 \%-3 \%$ ) in a small container, and anesthesia was maintained using a mixture of $100 \%$ oxygen and isoflurane $(1 \%-$ $2 \%$ ), administered through a small mask during MRI. Body temperature was monitored using a rectal temperature probe and maintained at $37^{\circ} \mathrm{C}$ using a heating blanket. Each rat was placed in the prone position within a surface coil. The ECG signal was obtained from three subcutaneous copper needles inserted in the left forelimb, hind limb, and right forelimb. The respiration signal was acquired from a respiratory pillow (SA Instruments Inc.) placed under the rat [9]. 
Scout imaging was initially performed using a gradient-echo sequence to localize the short-axis images at the middle level of the LV. Multi-slice cine images were obtained to cover the entire heart for functional analysis. After maintaining the respiratory rate at 30-50 cycles per minute by altering the isoflurane concentration, more than five (depending on the size of the heart) single-slice multi-echo T2*-mapping images were acquired on the short-axis slices during the mid-diastolic phase and end-inspiratory period using both ECG and respiratory gating systems. The imaging parameters were as follows: late gadolinium enhancement (LGE) imaging was performed by fast imaging with steady-state precession (FISP-cine on the same slice locations 10 min after the manual injection of gadolinium (Gd)diethylenetriamine penta-acetic acid (Magnevist, Bayer Health Care Pharmaceuticals, $0.15 \mathrm{mmol} / \mathrm{kg}$ ). Then, the same FISP-cine was repeated in identical planes after 2 min dobutamine (Gadovist, Bayer) injection at a rate of $10 \mathrm{mg} / \mathrm{kg} / \mathrm{min}$. Heart rate was measured before MR scan; before LDD injection; and 2,20 , and 30 min after LDD injection. Figure 1 shows the course of the examination.

The imaging parameters included: T2*-mapping: FA (Flip angle) $=30^{\circ}$,

$\mathrm{TR} / \mathrm{TE}=1000 \mathrm{~ms} / 3.5,7,10.5,14,17.5,21,24.5,28 \mathrm{~ms}$, Matrix size $=192 \times 192, \mathrm{FOV}=50 \times 50 \mathrm{~mm}$, and slice thickness $=1.5 \mathrm{~mm}$ without slice gap. LGE: $T R / T E=5.2 \mathrm{~ms} / 1.8 \mathrm{~ms}, F A=25^{\circ}$, matrix size $=256 \times 256$, $\mathrm{FOV}=50 \times 50 \mathrm{~mm}$, slice thickness $=1.5 \mathrm{~mm}, 25$ frames for each slice.

\section{Histology}

Following MRI evaluation, the rats were euthanized using potassium chloride ( $3 \mathrm{~mol} / \mathrm{L}, 0.5 \mathrm{~mL}$ ), and the hearts were rapidly excised. Each heart was cut into five or more transverse slices from the apex to the base; these slices measured approximately $1.5 \mathrm{~mm}$ in thickness to match the MRI slices. These slices were then incubated in $4 \%$ paraformaldehyde for hematoxylin and eosin staining.

\section{Data analysis}

The strain data were analyzed using commercially available software (Circle Cardiovascular Imaging, Inc.). The peak global radial strains (PGRS), peak global circumferential strains (PGCS), peak radial strains (PRS), and peak circumferential strains (PCS) were derived using an FT analysis of the LV shortaxis. The efficiency of PRS and PCS (EPRS and EPCS, respectively) were calculated using the following formula: PRS (PCS)/R-R interval. The analysis was based on the six segmentation created consisting of six hexahedral elements corresponding to the anteroseptal, anterior, anterolateral, posterolateral, posterior, and poster septal walls.

T2* maps were calculated using custom-made software written in Matlab 7.1 (The MathWorks, Inc.). More than five slices (slices with poor image quality due to motion artifacts were excluded from analysis) were selected to decide the regions of hemorrhage for each rat, which identified as a hypointense core within a hyper intense territory on T2* maps. In the sham group, the myocardium was segmented into six segments (Figure 2-3). 
The hyperenhanced myocardium on LGE images was defined as an $\mathrm{Ml}$ area; this was detected using a computer-aided threshold of $>5$ standard deviations (SDs) from the remote myocardium and adjusted manually and quantified using the multiple short-axis slice view. NMI areas were defined as segments without IMH and $\mathrm{MI}$ (The segments close to $\mathrm{MI}$ and IMH were exclusion for depressed strain compared with remote myocardium [11]. The MI and IMH distribution (segments) were evaluated by two experienced observers who were blinded to the experiment design. Discrepancies between the two observers were referred to another trained radiologist combined with the histopathologic results.

Normality was assessed using Kolmogorov-Smirnoff tests. Normally distributed data were expressed as mean \pm SD, and comparisons between the groups were conducted using analysis of variance.

Nonparametric data were expressed as median (25\%-75\% interquartile range) and compared using the Kruskal-Wallis test. $P$ values of $<0.05$ were considered statistically significant.

\section{Results}

\section{Animals}

MRI evaluations were successfully completed in 18 rats ( 9 in the reperfusion group and 9 in the sham group. Data from one rat was excluded because of death during surgery. Moreover, strain analysis was performed on 258 of 270 (95.6\%) of the segments in the sham group and 158 of 270 (58.5\%) of the segments of the reperfusion group $(\mathrm{IMH}=19 ; \mathrm{MI}=79$; and $\mathrm{NMI}=60)$. Table 1 shows the changes of the heart rate of the sham and reperfusion groups.

Table 1 The changes of heart rate of the sham group and reperfusion group

\begin{tabular}{|llllllr|}
\hline & $\begin{array}{l}\text { pre- } \\
\text { CMR/bpm }\end{array}$ & $\begin{array}{l}\text { pre- } \\
\text { LDD/bpm }\end{array}$ & $\begin{array}{l}\text { 2min post- } \\
\text { LDD/bpm }\end{array}$ & $\begin{array}{l}\text { 20min post- } \\
\text { LDD/bpm }\end{array}$ & $\begin{array}{l}\text { 30min post- } \\
\text { LDD/bpm }\end{array}$ & $P$ \\
\hline Sham group & $334 \pm 20$ & $261 \pm 13$ & $350 \pm 29$ & $324 \pm 14$ & $287 \pm 12$ & $<0.01$ \\
\hline $\begin{array}{l}\text { Reperfusion } \\
\text { group }\end{array}$ & $363 \pm 19$ & $269 \pm 31$ & $351 \pm 14$ & $332 \pm 23$ & $299 \pm 20$ & $<0.01$ \\
\hline
\end{tabular}

CMR, cardiovascular magnetic resonance; LDD, low-dose dobutamine

\section{The sham group}

The PGRS and PGCS increased after LDD injection in the sham group (Table 2). In addition, the segmental PRS and PCS increased after dobutamine injection, and the EPRS and EPCS increased (Table $3)$.

Table 2 The global strain parameters of the sham group and the reperfusion group 


\begin{tabular}{|lllllll|}
\hline & PGRS/\% & \multicolumn{5}{c|}{ PGCS $/ \%$} \\
\\
\hline rest & stress & P & rest & stress & P \\
\hline The sham group & $36.8 \pm 7.1$ & $46.8 \pm 8.6$ & $<0.05$ & $-19.6 \pm 2.4$ & $-22.0 \pm 2.7$ & $<0.05$ \\
\hline The reperfusion group & $35.7 \pm 8.1$ & $34.5 \pm 12.5$ & 0.734 & $-18.8 \pm 3.1$ & $-17.6 \pm 5.1$ & 0.337 \\
\hline
\end{tabular}

PGRS, peak global radial strains; PGCS, peak global circumferential strains

Table 3 The strain parameters of the sham group

\begin{tabular}{|c|c|c|c|c|c|c|}
\hline & \multicolumn{3}{|c|}{$\mathrm{PRS} / \%\left(\mathrm{M}\left(\mathrm{P}_{25}, \mathrm{P}_{75}\right)\right)$} & \multicolumn{3}{|c|}{$\mathrm{PCS} / \%\left(\mathrm{M}\left(\mathrm{P}_{25}, \mathrm{P}_{75}\right)\right)$} \\
\hline & rest & stress & $P$ & rest & stress & $\mathrm{P}$ \\
\hline strain & $\begin{array}{l}36.1 \\
\otimes 24.6,50.6 \rrbracket\end{array}$ & $50.4 \rrbracket 33.4,64 \rrbracket$ & $<0.01$ & $\begin{array}{l}-20.2 \\
\rrbracket-15.8 \rrbracket-23.9 \rrbracket\end{array}$ & $\begin{array}{l}-24 \\
\rrbracket-19.3 \rrbracket-26.5 \rrbracket\end{array}$ & $<0.01$ \\
\hline $\begin{array}{l}\text { The } \\
\text { efficiency of } \\
\text { strain }\end{array}$ & 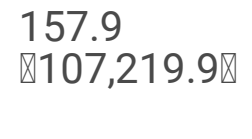 & 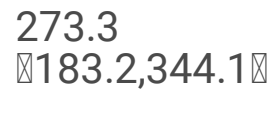 & $<0.01$ & $\begin{array}{l}-87.8 \\
\rrbracket-68.2 \rrbracket-104.4 \rrbracket\end{array}$ & $\begin{array}{l}-130 \\
\bigotimes-104.1 \rrbracket-143.2 \rrbracket\end{array}$ & $<0.01$ \\
\hline
\end{tabular}

PRS, peak radial strains; PCS, peak circumferential strains

\section{The reperfusion group}

The PGRS and PGCS did not increase after LDD injection in the reperfusion group (Table 2). The PRS and PCS of the IMH segment did not increase after dobutamine injection, and the EPRS and EPCS did not change (Table 4). Moreover, the PRS and PCS of the MI and NMI segments did not increase after dobutamine injection; however, the EPRS and EPCS increased (Table 5).

Table 4 Myocardial radial strains and circumferential strains in different segments of reperfusion group

\begin{tabular}{|c|c|c|c|c|c|c|}
\hline \multirow[b]{2}{*}{ Segment } & \multicolumn{3}{|c|}{$\mathrm{PRS} / \%\left(\mathrm{M}\left(\mathrm{P}_{25}, \mathrm{P}_{75}\right)\right)$} & \multicolumn{3}{|c|}{$\mathrm{PCS} / \%\left(\mathrm{M}\left(\mathrm{P}_{25}, \mathrm{P}_{75}\right)\right)$} \\
\hline & rest & stress & $\mathrm{P}$ & rest & stress & $P$ \\
\hline $\mathrm{IMH}$ & 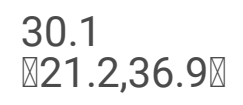 & 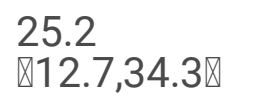 & 0.295 & $-16.8 \rrbracket-14 \rrbracket-19.1 \rrbracket$ & $\begin{array}{l}-14.6 \\
\rrbracket-9.7 \rrbracket-18.7 \rrbracket\end{array}$ & 0.198 \\
\hline MI & 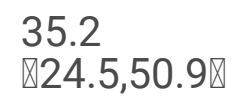 & $27.9 \llbracket 21,57.8 \rrbracket$ & 0.387 & $-19.4 \rrbracket-15 \rrbracket-24.2 \rrbracket$ & $\begin{array}{l}-17.1 \\
\rrbracket-13.9 \rrbracket-24.9 \rrbracket\end{array}$ & 0.171 \\
\hline NMI & $\begin{array}{l}39.2 \\
\otimes 21.7,64.1 \rrbracket\end{array}$ & 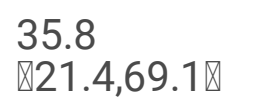 & 0.26 & $\begin{array}{l}-21.4 \\
\rrbracket-13.4 \rrbracket-26.8 \rrbracket\end{array}$ & $\begin{array}{l}-18.7 \\
\rrbracket-13.1 \rrbracket-26.6 \rrbracket\end{array}$ & 0.317 \\
\hline
\end{tabular}

PRS, peak radial strains; PCS, peak circumferential strains; IMH, intramyocardial hemorrhage; MI, myocardial infarction; NMI, non-myocardial infarction 
Table 5 The efficiency of radial strains and circumferential strains in different segments of reperfusion group

\begin{tabular}{|c|c|c|c|c|c|c|}
\hline \multirow[b]{2}{*}{ Segment } & \multicolumn{3}{|c|}{ EPRS $/ \% / s\left(M\left(P_{25}, P_{75}\right)\right)$} & \multicolumn{3}{|c|}{$\mathrm{EPCS} / \% / \mathrm{s}\left(\mathrm{M}\left(\mathrm{P}_{25}, \mathrm{P}_{75}\right)\right)$} \\
\hline & rest & stress & P & rest & stress & $P$ \\
\hline $\mathrm{IMH}$ & 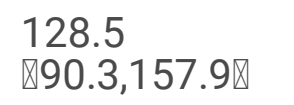 & $\begin{array}{l}132.3 \\
\otimes 75.2,184.8 \rrbracket\end{array}$ & 0.841 & $\begin{array}{l}-77.2 \\
\rrbracket-59.6 \rrbracket-84.8 \rrbracket\end{array}$ & $\begin{array}{l}-81.8 \\
\rrbracket-56.9 \rrbracket-100.4 \rrbracket\end{array}$ & 0.334 \\
\hline $\mathrm{Ml}$ & $\begin{array}{l}161.4 \\
\mathbb{1} 104.2,230.7 \rrbracket\end{array}$ & 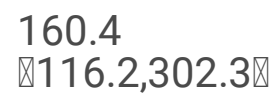 & $<0.05$ & $\begin{array}{l}-94.5 \\
\rrbracket-65.9 \bigotimes-117.2 \rrbracket\end{array}$ & $\begin{array}{l}-96.2 \\
\rrbracket-77.2 \rrbracket-135.5 \rrbracket\end{array}$ & $<0.05$ \\
\hline NMI & 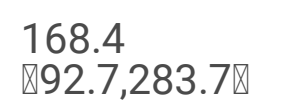 & 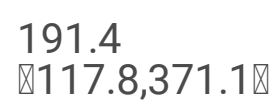 & $<0.05$ & $\begin{array}{l}-90.9 \\
\rrbracket-61.8 \rrbracket-117 \rrbracket\end{array}$ & $\begin{array}{l}-104.8 \\
\rrbracket-71.5 \rrbracket-144.6 \rrbracket\end{array}$ & $<0.01$ \\
\hline
\end{tabular}

EPRS, The efficiency of peak radial strains; EPCS, The efficiency of peak circumferential strains; IMH, intramyocardial hemorrhage; MI, myocardial infarction; NMI, non-myocardial infarction

The strains and efficiency of strain of the IMH segment were lower than those of the MI and NMI segments both before and after dobutamine injection (all $p<0.05$ ). No significant difference was observed between the strains and efficiency of strain of the Ml and NMI segments (all $p>0.05$ ).

\section{The differences between the two groups}

The strains and efficiency of strain of the IMH segment were lower than those of the sham group both before and after dobutamine injection (all $p<0.05$ ). Additionally, the PCS and EPCS of the MI segment were lower than those of the sham group both before and after dobutamine injection (all $p<0.05$ ). No significant differences in the PRS and EPRS were found between the MI segment and the sham group before dobutamine injection (both $p>0.05$ ), but differences were found after LDD injection (both $p<0.05)$. No significant differences in the strains and efficiency of strain were observed between the NMI segment and the sham group before dobutamine injection (both $p>0.05$ ); however, differences were found after LDD injection (both $p<0.05$ ).

\section{Histopathology}

Representative histopathologic images from animals with hemorrhagic infarctions sacrificed at 2nd are shown in Figure 3. H\&E stains easily showed evidence of myocardial injury (myocyte necrosis), the distribution of inflammatory cells and hemorrhage.

\section{Discussion}

This is one of a few studies using the CMR-FT technique in small animal models. We found that LDD would increase the PGRS and PGCS of normal rats, but not those of reperfused MI rats, considering that the heart rate, EPRS, and EPCS of the MI/NMI segments increased, whereas those of the IMH segment 
remained the same. Interestingly, no difference in the PRS and PCS was found between the MI and NMI segments.

Dobutamine significantly increased the heart rate and the maximum circumferential strain even at low doses [12], which improve cellular energetics and contractile function in the hypoperfused myocardium. LDD stress CMR has good specificity (83\%) and moderate sensitivity (74\%), similar to those of stress dobutamine echocardiography [13].

In this study, during anesthesia, the heart rate decreased from $310-380$ to $250-270 \mathrm{bpm}$, and dobutamine was helpful in recovering the heart rate to normal ranges; therefore, promoting the efficiency of myocardial strain except for the strain value in normal rats is beneficial. Furthermore, detecting the MI and NMI segments of the reperfusion group became more sensitive, whereas the strain remained the same after dobutamine injection.

Studies investigating the effect of IMH on myocardial function have reported that IMH is independently associated with poor prognosis and low global function. Limited data exist on the relationship between $\mathrm{IMH} /$ microvascular obstruction (MVO) and regional function. Henk Everaars et al. found that circumferential and radial strains were significantly diminished in segments with myocardial infarction and decreased even further if MVO was present [14]. Kidambi et al. studied the role of myocardial deformation using tissue tagging-derived strains in patients with acute reperfused MI [4]. They demonstrated that regional function is poor in myocardial segments with MVO and IMH. In an echo study, Zhao $\mathrm{H}$ et al. found that the segments of IMH had significantly decreased circumferential strains, but not longitudinal and radial strains [15].

Our study found that the PCS and EPCS of the MI segment were lower than those of the sham group without dobutamine stress, but not the PRS and EPRS. These differences can be explained in part by this way: among all strain parameters, circumferential strain was shown to be more accurate in assessing the infarct extension by identifying subtle impairments in LV contractile function.

In this study, the peak strain values reduced in the IMH segments, which conform to previous observations, and we further used LDD to find no changes in the IMH segments. LDD was used for CMR stress to discriminate between viable and nonviable myocardia and the segmental and global functional recovery of patients with ischemic cardiomyopathy [16]. We found that discriminating between IMH and non-IMH segments, especially with the efficiency of strain, was possible considering the heart rate.

Interestingly, we found no difference in the PRS and PCS between the MI and NMI segments during LDD stress. Two possible explanations exist for this result: First, the deterioration of the strains in the MI segments may affect not only the segment itself but also neighboring segments. Second, strain parameters describe myocardial deformation, which is also determined by passive movement.

Our study has some limitations. First, this study had a small sample size; however, a study demonstrated that myocardial strain parameters were highly reproducible even with a small number of animals. 
Therefore, no subgroups were set up for the MI segments according to the MI size. A larger sample size may be required to detect more subtle differences. Second, this study reported IMH only, not MVO; the reason was that FISP-cine with a relatively long acquisition time in the present study may underestimate the extent of MVO. Furthermore, the infusion method of LDD using veins was not performed in rats with small blood volumes [17]. Finally, we did not use cine in the long axis to calculate the longitudinal strain.

\section{Conclusions}

In conclusion, this study demonstrated a method in which LDD could be used in assessing the dysfunctions in segments with IMH, especially using the parameter of the efficiency of strain, which considers the heart rate. IMH could be a crucial factor that decreased segmental movement and reserved function to a greater extent, reducing the global function. A combination of LDD-CMR and LGE-CMR is a powerful tool for identifying which segment with impaired LV function will benefit from revascularization.

\section{Abbreviations}

IMH: Intramyocardial hemorrhage; MI: Myocardial infarction; LV: Left ventricular; LDD: Low-dose dobutamine; FT: Feature-tracking; NMI: Non-myocardial infarction; LGE: Late gadolinium enhancement; FISP Fast imaging with steady-state precession; PGRS: Peak global radial strains; PGCS: Peak global circumferential strains; PCS: Peak circumferential strains; PRS: Peak radial strains; SDs: Standard deviations; MVO: Microvascular obstruction; EPRS: The efficiency of peak radial strains; EPCS: The efficiency of peak circumferential strains.

\section{Declarations}

Ethics approval and consent to participate

All procedures performed in studies involving animals were in accordance with Guiding Principles for the Care and Use of Animals and was approved by the Animal Ethics Committee of West China Hospital, Sichuan University (Chengdu 610041) and the laboratory animal production license was 2021381A.

And this study was carried out in compliance with the Animal Research: Reporting in Vivo Experiments (ARRIVE) guidelines.

Consent for publication

Not applicable.

Availability of data and materials

All data generated or analysed during this study are included in this published article.

Competing interests

The authors declare that they have no competing interests 
Funding

This study was supported by The National Natural Science Foundation of China (81601546, 81520108014, 81771800, 81829003)

The role of the funder(s): conceived of the present idea in the design of the study.

Authors' contributions

Rui Xia, Yongmei Li, Fajin Lv and Fabao Gao designed the study.

Bo He, Tong Zhu, Yu Zhang, Yushu Chen and Jichun Liao collected and analyzed the MR data.

Yang Zhou analyzed and interpreted the histological data.

Rui Xia, Lei Wang and Jie Zheng optimized the MR sequences and wrote the manuscript.

All authors read and approved the final manuscript.

Acknowledgements

Not applicable

\section{References}

1. Galea N, Dacquino GM, Ammendola RM, Coco S, Agati L, De Luca L, et al. Microvascular obstruction extent predicts major adverse cardiovascular events in patients with acute myocardial infarction and preserved ejection fraction. Eur Radiol. 2019; 29:2369-2377.

2. Götte MJ, van Rossum AC, Twisk JWR, Kuijer JPA, Marcus JT, Visser CA. Quantification of regional contractile function after infarction: strain analysis superior to wall thickening analysis in discriminating infarct from remote myocardium. J Am Coll Cardiol. 2001; 37:808-817.

3. Hirsch A, Nijveldt R, Haeck JD, Beek AM, Koch KT, Henriques JP, et al. Relation between the assessment of microvascular injury by cardiovascular magnetic resonance and coronary Doppler flow velocity measurements in patients with acute anterior wall myocardial infarction. J Am Coll Cardiol. 2008; 51:2230-2238.

4. Kidambi A, Mather AN, Motwani M, Swoboda P, Uddin A, Greenwood JP, et al. The effect of microvascular obstruction and intramyocardial hemorrhage on contractile recovery in reperfused myocardial infarction: insights from cardiovascular magnetic resonance. J Cardiovasc Magn Reson. 2013; 15:58.

5. Bergerot C, Mewton N, Lacote-Roiron C, Ernande L, Ovize M, Croisille P, et al. Influence of microvascular obstruction on regional myocardial deformation in the acute phase of myocardial infarction: a speckle-tracking echocardiography study. J Am Soc Echocardiogr. 2014; 27:93-100.

6. Korosoglou G, Gitsioudis G, Voss A, Lehrke S, Riedle N, Buss SJ, et al. Strain-encoded cardiac magnetic resonance during high-dose dobutamine stress testing for the estimation of cardiac 
outcomes: comparison to clinical parameters and conventional wall motion readings. J Am Coll Cardiol. 2011; 58:1140-1149.

7. Glaveckaite S, Valeviciene N, Palionis D, Puronaite R, Serpytis P, Laucevicius A. Prediction of longterm segmental and global functional recovery of hibernating myocardium after revascularisation based on low dose dobutamine and late gadolinium enhancement cardiovascular magnetic resonance. J Cardiovasc Magn Reson. 2014; 16:83.

8. Lapinskas T, Grune J, Zamani SM, Jeuthe S, Messroghli D, Gebker R, et al. Cardiovascular magnetic resonance feature tracking in small animals - a preliminary study on reproducibility and sample size calculation. BMC Med Imaging. 2017; 17:51.

9. Xia R, Lu X, Zhang B, Wang Y, Liao J, Zheng J, et al. Assessment of myocardial edema and area at risk in a rat model of myocardial infarction with a faster T2 mapping method. Acta Radiol. 2015; 56:1085-1090.

10. Chen W, Zhang B, Xia R, Zhang R, Xu Z, Chen Y, et al. T2 mapping at 7T MRI can quantitatively assess intramyocardial hemorrhage in rats with acute reperfused myocardial infarction in vivo. $J$ Magn Reson Imaging. 2016; 44:194-203.

11. Kidambi A, Mather AN, Swoboda P, Motwani M, Fairbairn TA, Greenwood JP, et al. Relationship between myocardial edema and regional myocardial function after reperfused acute myocardial infarction: an MR imaging study. Radiology. 2013; 267:701-708.

12. Daire JL, Jacob JP, Hyacinthe JN, Croisille P, Montet-Abou K, Richter S, et al. Cine and tagged cardiovascular magnetic resonance imaging in normal rat at $1.5 \mathrm{~T}$ : a rest and stress study. $\mathrm{J}$ Cardiovasc Magn Reson. 2008; 10:48.

13. Bettencourt N, Chiribiri A, Schuster A, Nagel E. Assessment of myocardial ischemia and viability using cardiac magnetic resonance. Curr Heart Fail Rep. 2009; 6:142-153.

14. Everaars H, Robbers LFHJ, Götte M, Croisille P, Hirsch A, Teunissen PFA, et al. Strain analysis is superior to wall thickening in discriminating between infarcted myocardium with and without microvascular obstruction. Eur Radiol. 2018; 28:5171-5181.

15. Zhao H, Lee AP, Li Z, Qiao Z, Fan Y, An D, et al. Impact of Intramyocardial Hemorrhage and Microvascular Obstruction on Cardiac Mechanics in Reperfusion Injury: A Speckle-Tracking Echocardiographic Study. J Am Soc Echocardiogr. 2016; 29:973-982.

16. Bree D, Wollmuth JR, Cupps BP, Krock MD, Howells A, Rogers J, et al. Low-dose dobutamine tissuetagged magnetic resonance imaging with 3-dimensional strain analysis allows assessment of myocardial viability in patients with ischemic cardiomyopathy. Circulation. 2006; 114:I33-I36.

17. Nagel E, Lehmkuhl HB, Bocksch W, Klein C, Vogel U, Frantz E, et al. Noninvasive diagnosis of ischemia-induced wall motion abnormalities with the use of high-dose dobutamine stress MRI: comparison with dobutamine stress echocardiography. Circulation. 1999; 99:763-770.

\section{Figures}




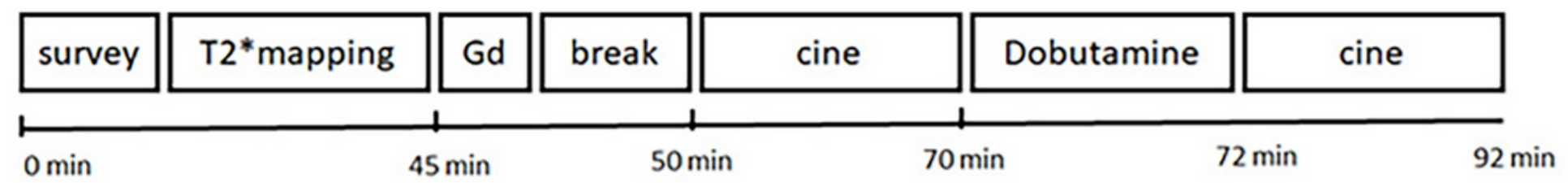

\section{Figure 1}

The time points of the examination.

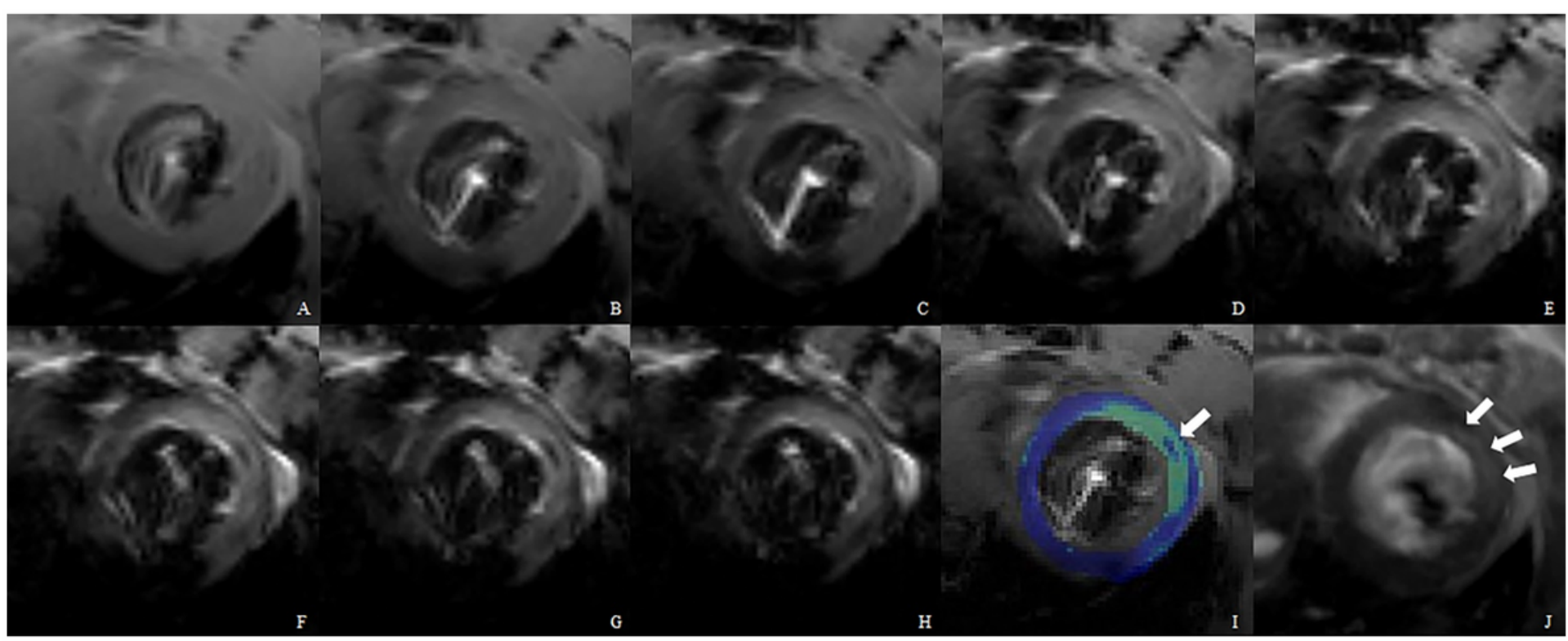

\section{Figure 2}

$\mathrm{IMH}, \mathrm{MI}$ areas showed by T2* images and LGE of MRI. A-H represent T2*weighted images while TE=3.5, $7,10.5,14,17.5,21,24.5,28 \mathrm{~ms}$, I demonstrated the IMH area (arrow) of T2* mapping sequence. J showed the myocardial infarction area (arrows) of LGE sequence. IMH, intramyocardial hemorrhage; MI, myocardial infarction; LGE, Late gadolinium enhancement. 


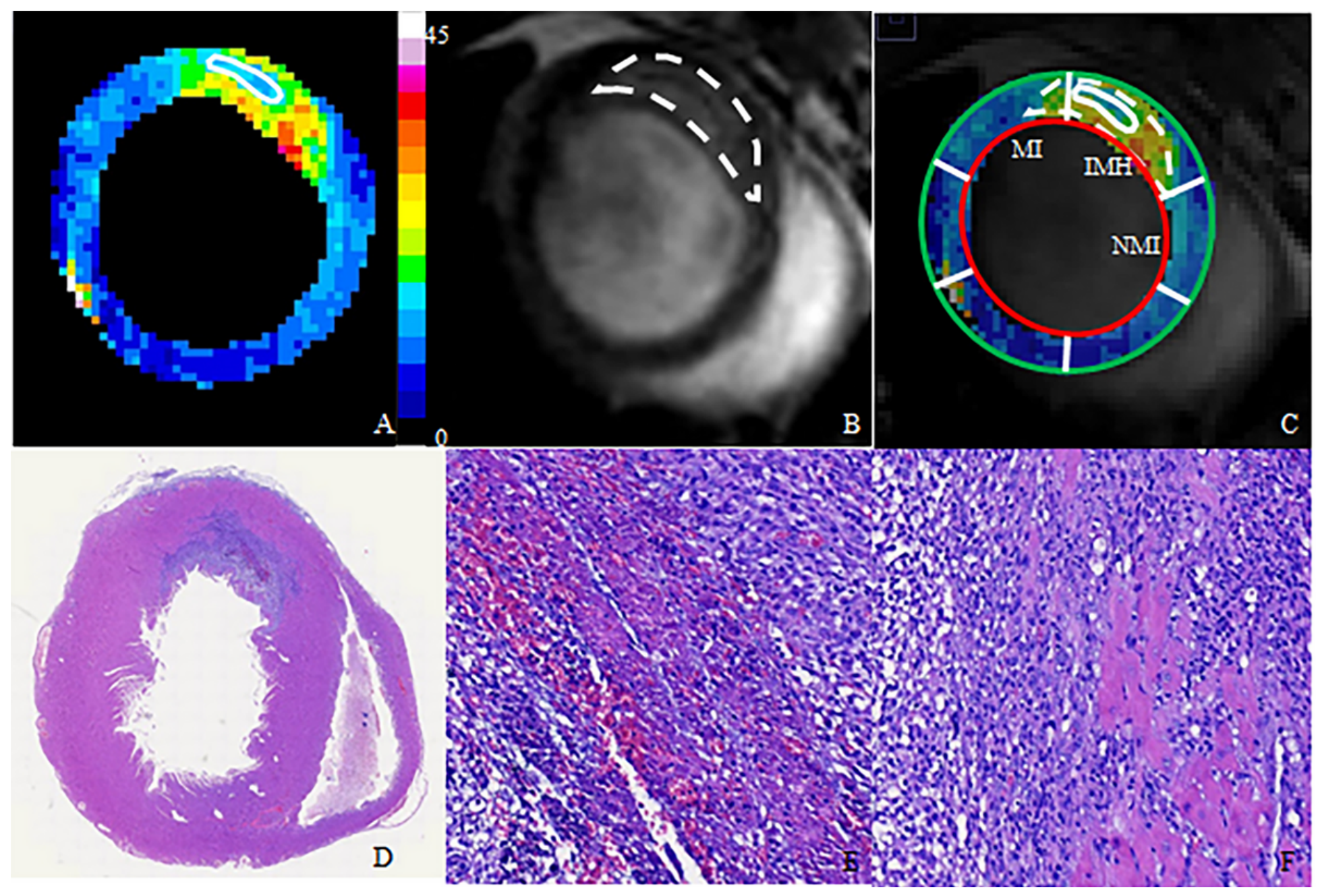

Figure 3

$\mathrm{IMH}, \mathrm{MI}$ and $\mathrm{NMI}$ areas showed by MRI and histological examination. (a) T2* mapping demonstrated the $\mathrm{IMH}$ area (white line). (b) LGE sequence showed the myocardial infarction area (white dotted line). (c) the overlay of $A$ and $B$ showed the IMH, MI and NMI segments which was used for strain analysis. (d) The examination of H\&E staining confirmed the incidence of IMH (e) and $\mathrm{MI}(\mathrm{f})$ which were magnified 20 in (e) and 20 in (f). IMH, intramyocardial hemorrhage; MI, myocardial infarction; NMI, no myocardial infarction; H\&E, hematoxylin and eosin. 